\title{
Flexible affine cones and flexible coverings
}

\author{
Mateusz Michałek $^{1,2,3}$ - Alexander Perepechko ${ }^{4,5}$. \\ Hendrik Süß ${ }^{6}$
}

Received: 3 December 2016 / Accepted: 19 February 2018 / Published online: 5 June 2018

(C) The Author(s) 2018

\begin{abstract}
We provide a new criterion for flexibility of affine cones over varieties covered by flexible affine varieties. We apply this criterion to prove flexibility of affine cones over secant varieties of Segre-Veronese embeddings and over certain Fano threefolds. We further prove flexibility of total coordinate spaces of Cox rings of del Pezzo surfaces.
\end{abstract}

Keywords Automorphism group · Transitivity · Flexibility · Affine cone · Cox ring · Segre-Veronese embedding $\cdot$ Secant variety $\cdot$ Del Pezzo surface

\section{Mathematics Subject Classification 14R20 - 14J50}

In this article we study affine algebraic varieties with the following property: the (special) automorphism group acts infinitely transitively on the regular locus. The systematic study of

The research of M. Michalek was supported by IP Grant 0301/IP3/2015/73 of the Polish Ministry of Science.

Mateusz Michałek

mmichalek@impan.pl

Alexander Perepechko

perepeal@gmail.com

Hendrik Süß

hendrik.suess@manchester.ac.uk

1 Mathematical Institut, Polish Academy of Sciences, Śniadeckich 8, 00-956 Warsaw, Poland

2 Freie Universität, Berlin, Germany

3 Max Planck Institute for Mathematics in the Sciences, Leipzig, Germany

4 Kharkevich Institute for Information Transmission Problems, 19 Bolshoy Karetny per., 127994 Moscow, Russia

5 Moscow Institute of Physics and Technology, 9 Institutskiy per., Dolgoprudny, 141701 Moscow Region, Russia

6 School of Mathematics, The University of Manchester, Alan Turing Building, Oxford Road, Manchester M13 9PL, UK 
this remarkable property and its complex analytic counterpart is presented in Arzhantsev et al. [1].

Let $X$ be an affine variety over an algebraically closed field $\mathbf{k}$ of characteristic zero. We consider actions of the additive group $\mathbb{G}_{a}=(\mathbf{k},+)$ on $X$. The subgroup of Aut $(X)$ generated by all $\mathbb{G}_{a}$-actions is called the special automorphism group of $X$ and will be denoted by $\operatorname{SAut}(X)$. We are interested in transitivity of the $\operatorname{SAut}(X)$-actions on the regular locus $X_{\text {reg }}$. Recall that an action of a group $G$ on a set $M$ is $m$-transitive if for every two $m$-tuples $\left(x_{1}, \ldots, x_{m}\right)$ and $\left(x_{1}^{\prime}, \ldots, x_{m}^{\prime}\right)$ of pairwise distinct elements of $M$ there exists $g \in G$ such that $g \cdot x_{i}=x_{i}^{\prime}$ for $i=1, \ldots m$.

We have the following result.

Theorem 0.1 ([1, Theorem 0.1]) Let $X$ be an irreducible affine variety of dimension $\geq 2$. Then the following conditions are equivalent.

(i) The group $\operatorname{SAut}(X)$ acts transitively on the regular locus $X_{\text {reg. }}$.

(ii) The group $\operatorname{SAut}(X)$ acts $m$-transitively on $X_{\text {reg }}$ for every $m>0$.

(iii) The tangent space of every $x \in X_{\text {reg }}$ is spanned by tangent vectors to orbits of $\mathbb{G}_{a^{-}}$ actions.

We say that $X$ is flexible if these conditions are fulfilled.

As examples of flexible varieties, let us mention affine cones over del Pezzo surfaces of degree $\geq 4$ (see [30]), over flag varieties, and affine toric varieties without torus factors [5]. It is also possible to construct new flexible varieties from a given flexible one, e.g. via suspensions [5] or open subsets with complement of codimension $\geq 2$ [14].

Our first main result provides a new criterion for flexibility of affine cones, see Sect. 1 for the proof. A similar, but independent, criterion using the notions of cylinders was provided in Perepechko [30, Th. 5].

Theorem 1.4 Let $Y$ be a normal projective variety covered by flexible affine open subsets $U_{i}, i \in I$, and $H$ be a very ample divisor on $Y$. If each subset $Y \backslash U_{i}$ is the support of an effective $\mathbb{Q}$-divisor $D_{i}$ linearly equivalent to $H$, then the affine cone $X=$ AffCone $_{H} Y$ is flexible.

Recall that a Segre-Veronese variety is an embedding of the direct product $\mathbb{P}^{d_{1}} \times \cdots \times \mathbb{P}^{d_{n}}$ of projective spaces by the very ample line bundle of the form $\mathcal{O}\left(s_{1}\right) \otimes \cdots \otimes \mathcal{O}\left(s_{n}\right)$. Further, given a projective variety $X \subset \mathbb{P}^{n}$, the Zariski closure of the union of the secant (resp. tangent) lines to $X$ is called a secant (resp.tangential) variety of $X$. As the first application of Theorem 1.4, we deduce the following result, see Sect. 2 for details.

Theorem 2.20 Let $X=v_{s_{1}}\left(\mathbb{P}\left(V_{1}\right)\right) \times \cdots \times v_{s_{n}}\left(\mathbb{P}\left(V_{n}\right)\right)$ be a Segre-Veronese variety. Then the affine cone over the secant variety of $X$ is flexible. Further, if $s_{1}=\cdots=s_{n}=1$, then also the affine cone over the tangential variety of $X$ is flexible.

Our proof technique relies on triangular transformations of the affine charts of the ambient projective space. They are inspired by algebraic statistics, precisely by computation of cumulants $[10,29,34,41,42]$.

Section 3 contains preliminaries on smooth rational $T$-varieties of complexity one. These are varieties $X$ with an effective action of a torus $T$, where $\operatorname{dim} X=\operatorname{dim} T+1$. Section 4 is devoted to flexibility of affine cones over such varieties.

In Arzhantsev et al. [6] it was shown that smooth varieties of this type admit a toric covering and for certain affine cones over these varieties we, indeed, obtain flexibility. For example, this applies to all known Fano threefolds with 2-torus action. We use below the list of Fano threefolds in Mori-Mukai's classification [26]. 
Theorem 4.5 All the affine cones over the Fano threefolds $Q, 2.29,2.30,2.31,2.32,3.8$, $3.18,3.19,3.23,3.24,4.4$, and certain elements of the families 2.24, 3.10 admitting a 2-torus action in Mori-Mukai's classification are flexible.

The main tool to obtain these results is the combinatorial description of $T$-varieties developed in Altmann and Hausen [2] and Altmann et al. [3], which in the case of complexity one allows to study (torus equivariant) coverings as in Theorem 1.4.

While Theorems 2.20 and 4.5 are concerned with projective coordinate rings, in Sect. 5 of the paper we obtain related results for total coordinate rings or Cox rings.

Theorem 5.4 The total coordinate spaces of smooth del Pezzo surfaces are flexible.

This was known so far only for the toric del Pezzo surfaces (i.e. those of degree 9, 8, 7 and 6) and by Arzhantsev et al. [5, Thm. 0.2] for the case of degree 5, where the total coordinate space is known to be the affine cone over the Grassmannian $G(2,5)$. On the other hand, this extends a result of Arzhantsev et al. [6], where flexibility was proved only outside a subset of codimension 2 .

Theorem 5.9 The total coordinate space of a complete smooth rational T-variety of complexity one is flexible.

\section{Flexibility of affine cones}

Lemma 1.1 Let $X \subset \mathbb{A}^{n+1}$ be the affine cone over a projective variety $Y \subset \mathbb{P}^{n}$ of dimension $\geq 1$. Consider a subgroup $G \subset$ Aut $X$ such that

- the canonical $\mathbb{G}_{m}$-action on $X$ by homotheties sends $G$-orbits to G-orbits,

- all $G$-orbits are locally closed, and

- there is an orbit $G x \subset X \backslash\{0\}$, whose image $Y^{*}$ under the projection $\pi: X \backslash\{0\} \rightarrow Y$ is an open subset in $Y$ with complement of codimension $\geq 2$.

Then $G x=\pi^{-1}\left(Y^{*}\right)$ and is open in $X$.

Proof Since the $G$-action is $\mathbb{G}_{m}$-equivariant, $X^{*}=\pi^{-1}\left(Y^{*}\right)$ is a union of $G$-orbits, whose projections coincide with $Y^{*}$. Hence $X^{*}=\bigcup_{\lambda \in \mathbb{G}_{m}} \lambda G x$, where all $G$-orbits are closed in $X^{*}$.

Let us show that $G x=X^{*}$. Assume the contrary. Then $\operatorname{dim} G x=\operatorname{dim} Y$ and the stabilizer $S \subset \mathbb{G}_{m}$ of the orbit $G x$ is finite. Indeed, two points $v, v^{\prime} \in G x$ lie in the same $\mathbb{G}_{m}$-orbit if and only if $v=\lambda \cdot v^{\prime}$ for some $\lambda \in \mathbb{G}_{m}$. The latter is equivalent to $\lambda G x=G x$, i.e., $\lambda \in S$. So, $G v \cap \mathbb{G}_{m} v=S v$ for any $v \in X^{*}$.

Denote by $X^{\times}$the blow up of $X$ at 0 . This is the total space of the line bundle $\mathcal{O}_{Y}(-1)$ over $Y$. Consider the quotient morphism

$$
\mu: X^{\times} \stackrel{/ S}{\longrightarrow} X^{\prime}=X^{\times} / S
$$

given by $t \mapsto t^{|S|}$ on every trivialization chart, where $t$ is the coordinate of the fibers. Then $\mu(G x)$ intersects each fiber at most once, so it is a meromorphic, nonvanishing section of the line bundle $X^{\prime} \rightarrow Y$. Indeed, it is a graph of a rational function on trivialization charts of the line bundle. However, the subset $D \subset Y$, where our rational section of the line bundle $X^{\prime} \rightarrow Y$ is not defined or vanishes, if non-empty, is a Cartier divisor. 
On the other hand, under our assumptions $D \subseteq Y \backslash Y^{*}$ is of codimension at least 2 . Therefore, $D$ is empty and $\mu(G x)$ is a global section of $X^{\prime} \rightarrow Y$ disjoint with the zerosection. Since $Y$ is of positive dimension $X^{\prime} \rightarrow Y$ is non-trivial. This gives a contradiction.

So, the group $G$ acts on $X^{*}$ transitively.

Corollary 1.2 Under the setup of Lemma 1.1, if $Y$ is smooth in codimension one and $\pi(G x)=Y^{*}$ coincides with the regular locus $Y_{\mathrm{reg}}$, then $G x=X_{\mathrm{reg}} \backslash\{0\}$.

Lemma 1.3 Let $Y \subset \mathbb{P}^{n}$ be a linearly nondegenerate projective variety, and let $H=Y \cap$ $\left\{x_{0}=0\right\}$ be a hyperplane section of $Y$. Suppose that $U=Y \backslash H$ is endowed with a $\mathbb{G}_{a^{-}}$ action $\phi: \mathbb{G}_{a} \times U \rightarrow U$. Let $\pi: X \backslash\left\{x_{0}=0\right\} \rightarrow U$ be the natural projection, where $X=$ AffCone $Y \subset \mathbb{A}^{n+1}$ is the affine cone over $Y$. Then $X$ admits a $\mathbb{G}_{a}$-action $\hat{\phi:} \mathbb{G}_{a} \times X \rightarrow X$ normalised by the $\mathbb{G}_{m}$-action of the cone, such that

- $\phi\left(\mathbb{G}_{a} \times\{\pi(x)\}\right)=\pi\left(\hat{\phi}\left(\mathbb{G}_{a} \times\{x\}\right)\right)$ for any $x \in \pi^{-1}(U)$.

- $\hat{\phi}$ is trivial on $X \backslash \pi^{-1}(U)$.

In other words, $\pi: X \backslash\left\{x_{0}=0\right\} \rightarrow U$ provides a correspondence between $\hat{\phi}$-orbits and $\phi$-orbits.

Proof Let $Y$ be defined by a homogeneous ideal $I \subset \mathbf{k}\left[x_{0}, \ldots, x_{n}\right]$ which does not contain $x_{0}, \mathbf{k}[X]=\mathbf{k}\left[x_{0}, \ldots, x_{n}\right] / I$, and $U=\left\{x_{0} \neq 0\right\} \subset Y$.

There exists a natural embedding $\rho: U \hookrightarrow X, \rho(U)=\left\{x_{0}=1\right\} \subset X$. On the other hand, $\pi: X \backslash\left\{x_{0}=0\right\} \rightarrow U$ is a trivial $\mathbb{G}_{m}$-bundle. Therefore, we may extend the $\mathbb{G}_{a}$-action $\phi$ on $U$ to a $\mathbb{G}_{a}$-action $\tilde{\phi}$ on $X \backslash\left\{x_{0}=0\right\}$ defined by a homogeneous locally nilpotent derivation $\tilde{\delta}$.

There exists $d \in \mathbb{N}$ such that $x_{0}^{d} \tilde{\delta}\left(x_{i}\right) \in \mathbf{k}[X]$ for $i=1, \ldots, n$. Since $x_{0} \in \operatorname{ker} \tilde{\delta}$, a homogeneous derivation $\hat{\delta}=x_{0}^{d+1} \tilde{\delta}$ on $X$ is locally nilpotent. The corresponding $\mathbb{G}_{a}$-action $\hat{\phi}$ is normalised by the $\mathbb{G}_{m}$-action, coincides with $\phi$ on the hypeplane section $\left\{x_{0}=1\right\} \cong U$ of $X$, and is trivial on $\left\{x_{0}=0\right\} \subset X$.

Theorem 1.4 Let $Y$ be a normal projective variety covered by flexible affine open subsets $U_{i}, i \in I$, and $H$ be a very ample divisor on $Y$. If each subset $Y \backslash U_{i}$ is the support of an effective $\mathbb{Q}$-divisor $D_{i}$ linearly equivalent to $H$, then the affine cone $X=\operatorname{AffCone}_{H} Y$ is flexible.

Proof The tangent space at 0 to $X$ must contain all lines through 0 contained in $X$. As $X$ is covered by such lines, the tangent space is equal to the linear span of $X$. If $X=\mathbb{A}^{n+1}$ the theorem is trivial. Otherwise, the origin is a singular point of $X$, which we assume from now on.

For each subset $U_{i}$ there exists a finite number of $\mathbb{G}_{a}$-actions $\left\{\phi_{i j}\right\}$ such that the orbit of the group generated by them is the regular locus of $U_{i}$, see [1, Prop. 1.5]. Let $k \in \mathbb{N}$ be such that $k D_{i}$ is a $\mathbb{Z}$-divisor for any $i \in I$. For each action $\phi_{i j}$ we can consider a lifted action $\tilde{\phi}_{i j}$ on AffCone $_{k H} Y$ as in Lemma 1.3. Since the Veronese map $X \rightarrow$ AffCone $_{k H} Y$ is unramified outside the vertex, Theorem 1.3 of Masuda and Miyanishi [27] implies the existence of an action $\hat{\phi}_{i j}$ on $X$, whose orbits have the same image in $Y$ as the orbits of $\tilde{\phi}_{i j}$.

Let a subgroup $G=\left\langle\hat{\phi}_{i j}\right\rangle \subset$ SAut $X$ be generated by the $\mathbb{G}_{a}$-actions on $X$ which correspond to all the open subsets $U_{i}$. Then the image of the orbit $G x$ of a regular point $x \in X_{\text {reg }}$

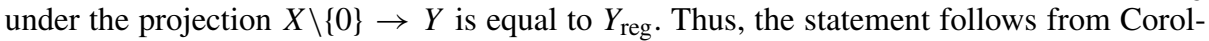
lary 1.2, as the variety $Y$ is normal, in particular smooth in codimension one, and the $G$-orbits are locally closed by Arzhantsev et al. [1, Prop. 1.3]. 
Example 1.5 Consider $\mathbb{P}^{n}$ with coordinates $x_{0}, \ldots, x_{n}$ and a smooth subvariety $Y=$ $\mathbb{V}\left(x_{0}, f\right)$ of codimension 2, where $f$ is an irreducible homogeneous polynomial of degree $d$. Let $q: X \rightarrow \mathbb{P}^{n}$ be the blowup in $Y$. We apply Theorem 1.4 to show that all the affine cones over $X$ are flexible. Notice that $X$ is a Fano variety if $d \leq n$ holds.

Let $U_{i}:=\mathbb{P}^{n} \backslash H_{i}:=\mathbb{P}^{n} \backslash \mathbb{V}\left(x_{i}\right)$. The preimage $q^{-1}\left(U_{0}\right)$ is isomorphic to $U_{0} \cong \mathbb{A}^{n}$, since $Y$ does not intersect $U_{0}$. For $i \neq 0$ the preimage $q^{-1}\left(U_{i}\right)$ is given by

$$
V\left(\frac{f}{x_{i}^{d}} \cdot u-\frac{x_{0}}{x_{i}} \cdot v\right) \subset U_{i} \times \mathbb{P}^{1} .
$$

Hence, $q^{-1}\left(U_{i}\right)$ is covered by the affine charts

$$
U_{i}^{0}:=q^{-1}\left(U_{i}\right) \backslash[v=0] \quad \text { and } \quad U_{i}^{\infty}:=q^{-1}\left(U_{i}\right) \backslash[u=0],
$$

the first one being an affine space and the second one being isomorphic to

$$
V\left(\frac{f}{x_{i}^{d}}-\frac{x_{0}}{x_{i}} \cdot \frac{v}{u}\right) \subset \operatorname{Spec} k\left[\frac{x_{0}}{x_{i}}, \ldots, \frac{x_{n}}{x_{i}}, \frac{v}{u}\right] .
$$

In the notation of Arzhantsev et al. [5] this is a suspension over $\mathbb{A}^{n-1}$ and hence flexible by Theorem 0.2 in loc. cit.

Given a divisor $D \subset \mathbb{P}^{n}$, we denote by $\widetilde{D}$ its strict transform on the blowup. We also denote by $E$ the exceptional divisor at $Y$ and by $H$ the pullback of a general hyperplane. To see that the given covering is polar ([22, Def. 3.7]) with respect to every ample divisor, note that

$$
X \backslash q^{-1}\left(U_{0}\right)=E \cup \widetilde{H}_{0} ; X \backslash U_{i}^{\infty}=\widetilde{H}_{i} \cup \widetilde{H}_{0} ; X \backslash U_{i}^{0}=\widetilde{H}_{i} \cup \widetilde{\mathbb{V}(f)} ; \text { for } i>0 .
$$

Now, $X$ has Picard group $\mathbb{Z}^{2}$ with generators $[H]$ and $[E]$. The effective cone is generated by $[E]$ and $[H]-[E]$ and the nef-cone by $[H]$ and $d[H]-[E]$. Moreover, $[\widetilde{\mathbb{V}(f)}]=$ $d[H]-[E]$ and $\left[\widetilde{H}_{0}\right]=[H]-[E]$ hold. So, for every affine charts from (1) the boundary components in (2) span a cone in the Neron-Severi space containing the whole nef cone of $X$. Hence, every ample class can be expressed as a positive linear combination of the complement components. In other words, for every affine charts $U$ from (1) there is an effective divisor $D$ with support $X \backslash U$, which lies in the chosen ample class. By Theorem 1.4 the flexibility of the corresponding affine cone follows.

Let further be $q^{\prime}: X^{\prime} \rightarrow \mathbb{P}^{n}$ the combined blowup of $\mathbb{P}^{n}$ in $Y$ as above and additionally in the point $y=(1: 0: \cdots: 0)$. Similarly, using the same notation and denoting by $E^{\prime}$ the exceptional divisor of the blowup in the point $y$, we have the following flexible charts on $X^{\prime}$ :

$$
U_{0}^{i}:=X \backslash\left(\widetilde{H}_{0} \cup E \cup \widetilde{H}_{i}\right), \quad U_{i}^{\infty}:=X \backslash\left(\widetilde{H}_{i} \cup \widetilde{H}_{0} \cup E^{\prime}\right), \quad U_{i}^{0}:=X \backslash\left(\widetilde{H}_{i} \cup \widetilde{\mathbb{V}(f)} \cup E^{\prime}\right) .
$$

The first two are affine spaces and the last one is a suspension as before. We see that the complements of the affine charts always consist of three components with classes corresponding to one of the triples

$$
\begin{aligned}
& \left([H]-[E],[E],[H]-\left[E^{\prime}\right]\right),\left([H]-\left[E^{\prime}\right], d[H]-[E],\left[E^{\prime}\right]\right), \\
& \left([H]-\left[E^{\prime}\right],[H]-[E],\left[E^{\prime}\right]\right) .
\end{aligned}
$$

Further, each triple spans a cone containing the nef cone of $X^{\prime}$, which is spanned by $d[H]-[E],[H]-\left[E^{\prime}\right]$, and $[H]$. Hence, every ample class can be expressed as a positive linear combination of complement components. As above this implies the flexibility of the corresponding affine cone by Theorem 1.4. 


\section{Secant of Segre-Veronese variety}

This section is based on Michalek et al. [29], where the toric covering of a Segre variety was constructed. Here we generalize that construction to a Segre-Veronese variety and hence prove Theorem 2.20. Throughout this section by a parameterization of a variety $Z$ we mean a dominant morphism from a dense open subset of an affine space to $Z$.

Definition 2.1 Given for each $i=1, \ldots, n$ a finite-dimensional vector space $V_{i}$ and its symmetric power $S^{S_{i}}\left(V_{i}\right), s_{i} \in \mathbb{Z}_{>0}$, the Segre-Veronese variety

$$
X=v_{s_{1}}\left(\mathbb{P}\left(V_{1}\right)\right) \times \cdots \times v_{s_{n}}\left(\mathbb{P}\left(V_{n}\right)\right) \subset \mathbb{P}\left(S^{S_{1}}\left(V_{1}\right) \otimes \cdots \otimes S^{S_{n}}\left(V_{n}\right)\right)
$$

is defined as the embedding of the product $\mathbb{P}\left(V_{1}\right) \times \cdots \times \mathbb{P}\left(V_{n}\right)$ by the very ample line bundle $\mathcal{O}\left(s_{1}\right) \otimes \cdots \otimes \mathcal{O}\left(s_{n}\right)$.

We will be using an equivalent construction. Apart from (projective) Segre-Veronese varieties we will consider affine cones over them and refer to those as Segre-Veronese cones. They should not be confused with intersections of Segre-Veronese varieties with principal affine open subsets, which also play a crucial role.

For each $V_{i}, 1 \leq i \leq n$, we denote $d_{i}=\operatorname{dim} V_{i}-1$ and fix a basis $e_{0}^{i}, \ldots, e_{d_{i}}^{i}$ of $V_{i}$. We also denote elements of the basis of $V_{i}^{\otimes s}$ by

$$
e_{i_{1}, \ldots, i_{s}}^{i}=e_{i_{1}}^{i} \otimes \cdots \otimes e_{i_{s}}^{i} .
$$

Thus, the symmetric power is $S^{s_{i}}\left(V_{i}\right)=$

$$
\left\{v \in V_{i}^{\otimes s} \mid\left(e_{i_{1}, \ldots, i_{s}}^{i}\right)^{*}(v)=\left(e_{i_{\sigma(1)}, \ldots, i_{\sigma(s)}}^{i}\right)^{*}(v) \text { for any permutation } \sigma \in S_{s}\right\} .
$$

This allows us to embed the Veronese cone into the Segre cone and obtain the following diagram:

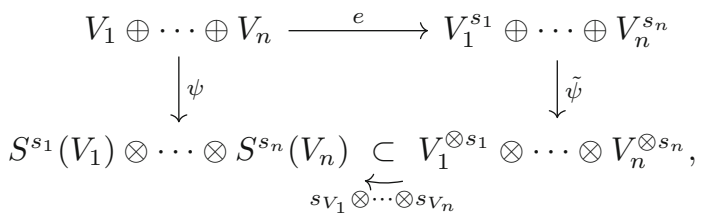

where

- $e:\left(v_{1}, \ldots, v_{n}\right) \mapsto\left(v_{1}, \ldots, v_{1}, v_{2}, \ldots, v_{2}, v_{3}, \ldots, v_{n}\right)$ is the diagonal embedding,

- $\tilde{\psi}:\left(v_{1}^{1}, \ldots, v_{1}^{s_{1}}, v_{2}^{1}, \ldots, v_{2}^{s_{2}}, v_{3}^{1}, \ldots, v_{n}^{s_{n}}\right) \mapsto v_{1}^{1} \otimes \cdots \otimes v_{n}^{s_{n}}$ is the parameterization of the Segre cone, which is a nonlinear map,

- $\psi=\left.\tilde{\psi}\right|_{\operatorname{im}(e)} \circ e$ is the parameterization of the Segre-Veronese cone, and

- $s_{V_{i}}: V_{i}^{\otimes s_{i}} \rightarrow S^{s_{i}}\left(V_{i}\right)$ are the natural symmetrizing projections.

Notation 2.2 (i) For a vector space $V=V_{i_{1}} \otimes \cdots \otimes V_{i_{k}}$ we denote

$$
\hat{V}:=\left\{x \in V \mid\left(e_{0}^{i_{1}} \otimes \cdots \otimes e_{0}^{i_{k}}\right)^{*}(x)=1\right\}
$$

and regard it as a vector space with basis $\left\{e_{j_{1}}^{i_{1}} \otimes \cdots \otimes e_{j_{k}}^{i_{k}} \mid j_{1}+\cdots+j_{n}>0\right\}$.

(ii) We may, and often will, consider $\hat{V}$ as a complement to a hyperplane section $\{[x]$ : $\left.\left(e_{0}^{i_{1}} \otimes \cdots \otimes e_{0}^{i_{k}}\right)^{*}(x)=0\right\}$ of $\mathbb{P}(V)$. Thus $\hat{V}$ may be regarded as an affine open subset of $\mathbb{P}(V)$. 
(iii) We denote $A:=V_{1}^{\otimes s_{1}} \otimes \cdots \otimes V_{n}^{\otimes s_{n}}$.

(iv) We also define

$$
\begin{aligned}
& B:=\prod_{i=1}^{n} \hat{V}_{i}{ }^{\times s_{i}} \subset V_{1}^{s_{1}} \oplus \cdots \oplus V_{n}^{s_{n}}, \\
& B^{\prime}:=\prod_{i=1}^{n} \hat{V}_{i} \subset V_{1} \oplus \cdots \oplus V_{n} .
\end{aligned}
$$

(v) Finally, we denote $\pi: A \backslash\{0\} \rightarrow \mathbb{P}(A)$ and obtain the following diagram of open subsets:

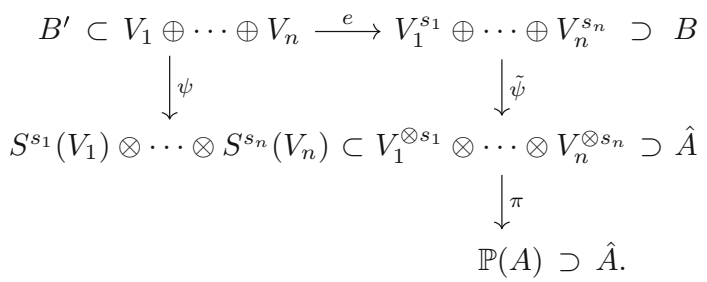

Remark 2.3 Since $\mathbb{P}\left(S^{s_{1}}\left(V_{1}\right) \otimes \cdots \otimes S^{s_{n}}\left(V_{n}\right)\right) \subset \mathbb{P}(A)$, we can study the Segre-Veronese variety as a subvariety of $X=\overline{\pi \circ \psi\left(B^{\prime}\right)} \subset \mathbb{P}(A)$. Note that the image of $\left.\tilde{\psi}\right|_{B}$ does not contain the origin.

\section{Cumulants}

In this setting we may apply the (nonlinear) coordinate systems of $B$, called cumulants and presented in Michalek et al. [29]. For the motivations to consider them, coming from algebraic statistics, we refer the reader to Refs. [34,41,42]. A general mathematical setting for these methods is well presented in Ciliberto et al. [10]. Further results are obtained for other varieties, e.g. Grassmannians and spinor varieties [28]. However, in other cases we do not obtain toric coverings. Still, we believe that similar methods can be applied to a larger class of secant and tangential varieties.

Notation 2.4 Basis elements of $A$ are of the form $e_{c_{1}^{1}, \ldots, c_{s_{1}}^{1}}^{1} \otimes \cdots \otimes e_{c_{1}^{n}, \ldots, c_{s_{n}}^{n}}^{n}$ and are in natural correspondence with tuples $\left(c_{1}^{1}, \ldots, c_{s_{1}}^{1}, \ldots, c_{1}^{n}, \ldots, c_{s_{n}}^{n}\right)$, where $0 \leq c_{j}^{i} \leq d_{i}$ for $1 \leq i \leq n$ and $1 \leq j \leq s_{i}$. Let us denote the set of these tuples by $C(A)$ and for each $c \in C(A)$ the corresponding basis element by $e(c)$. Finally, denote the dual basis elements by $x(c)=e(c)^{*}$. Similarly, we denote $C(\hat{A})=C(A) \backslash\{(0, \ldots, 0)\}$.

Definition 2.5 (degree, ordering) Given a tuple $c \in C(\hat{A})$, the number of its nonzero entries is called the degree of $c$. Given $c_{1}, c_{2} \in C(\hat{A})$, we say that $c_{1} \leq c_{2}$ if $c_{1}$ can be obtained from $c_{2}$ by setting some entries to zero.

Thus, we have a natural poset structure on $C(\hat{A})$, which induces a poset structure on the basis of $\hat{A}$. Simply speaking, the ordering on the basis is defined by replacing $e_{j}^{i}$ by $e_{0}^{i}$ in the tensor product elements.

Example 2.6 Consider $t_{2}:=e_{1}^{1} \otimes e_{0}^{1} \otimes e_{5}^{2}, t_{1}:=e_{0}^{1} \otimes e_{0}^{1} \otimes e_{5}^{2}$ and $t_{0}:=e_{1}^{1} \otimes e_{3}^{1} \otimes e_{4}^{2}$. We have $t_{1}<t_{2}$ and $t_{1}, t_{2}$ are not comparable with $t_{0}$. 
Denote the set of indices

$$
\operatorname{Ind}(\hat{A})=\left\{\left[\begin{array}{l}
1 \\
1
\end{array}\right], \ldots,\left[\begin{array}{l}
1 \\
s_{1}
\end{array}\right], \ldots,\left[\begin{array}{c}
n \\
1
\end{array}\right], \ldots,\left[\begin{array}{c}
n \\
s_{n}
\end{array}\right]\right\}
$$

and endow it with a natural lexicographic order, namely, the order of appearance above. Given $c \in C(\hat{A})$ and a subset of indices $I \subset \operatorname{Ind}(\hat{A})$, we introduce the index tuple

$$
c_{I}=\left(b_{1}^{1}, \ldots, b_{s_{n}}^{n}\right), \quad \text { where } b_{j}^{i}=\left[\begin{array}{cc}
c_{j}^{i}, & {\left[\begin{array}{l}
i \\
j
\end{array}\right] \in I,} \\
0, & {\left[\begin{array}{l}
i \\
j
\end{array}\right] \notin I .}
\end{array}\right.
$$

Note that $\{b \in C(\hat{A}) \mid b \leq c\}=\left\{c_{I} \mid I \subset \operatorname{Ind}(\hat{A})\right\}$. We will use either one-element subsets $I=\left[\begin{array}{l}i \\ j\end{array}\right]$ or subsets of the following form, where $i_{1}, i_{2} \in \operatorname{Ind}(\hat{A})$ :

$$
I=\left[i_{1}: i_{2}\right]:=\left\{i \mid i_{1} \leq i<i_{2}\right\} \subset \operatorname{Ind}(\hat{A}) .
$$

Definition 2.7 A thick interval partition of a tuple $c \in C(\hat{A})$ of degree at least two is an increasing sequence of indices $\left[\begin{array}{l}1 \\ 1\end{array}\right]=b_{0}<\ldots<b_{k}=\left[\begin{array}{c}n \\ s_{n}\end{array}\right]$ such that $\operatorname{deg} c_{\left[b_{i}: b_{i+1}\right]} \geq 2$ for each $i$. The set of all thick interval partitions of $c$ will be denoted by $I P(c)$. It is always nonempty as it contains $\left\{\left[\begin{array}{l}1 \\ 1\end{array}\right],\left[\begin{array}{c}n \\ s_{n}\end{array}\right]\right\}$.

Now we can recall the coordinate systems from [29, Sec. 2].

Notation 2.8 For each $c \in C(\hat{A})$ we denote

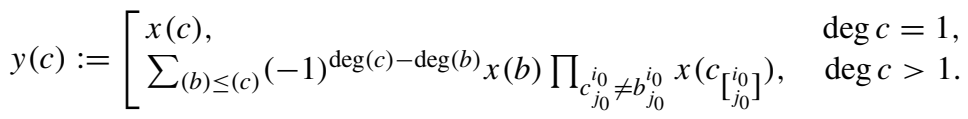

Then for each $c \in C(\hat{A})$ we introduce a function in $\mathbf{k}[\hat{A}]$

$$
z(c):=\left[\begin{array}{ll}
y(c), & \operatorname{deg} c=1, \\
\sum_{\left(b_{0}, \ldots, b_{k}\right) \in I P(c)}(-1)^{k} \prod_{m=1}^{k} y\left(c_{\left[b_{m-1}: b_{m}\right]}\right), & \operatorname{deg} c>1 .
\end{array}\right.
$$

Lemma 2.9 Each one of the sets $\{x(c)\}_{c \in C(\hat{A})},\{y(c)\}_{c \in C(\hat{A})}$, and $\{z(c)\}_{c \in C(\hat{A})}$ is an algebraically independent system of functions generating $\mathcal{O}(\hat{A})$. In other words, $\{z(c)\}$ is a coordinate system on $\hat{A}$ as an affine space.

Proof Since $y(c)$ is a sum of $x(c)$ and of terms of smaller degree, the endomorphism of $\mathbf{k}[\hat{A}]$ that maps $x(c)$ to $y(c)$ for each $c$ is invertible. The same holds for $\{y(c)\}$ and $\{z(c)\}$. So, the statement follows.

\section{Secant}

The secant variety $\operatorname{Sec} X \subset \mathbb{P}(A)$ of the Segre-Veronese variety $X$ is parameterized by a map

$$
\sec _{X}: \mathbb{A}^{1} \times B^{\prime} \times B^{\prime} \rightarrow \hat{A}, \quad(t, v, w) \mapsto \pi(t \cdot \psi(v)+(1-t) \cdot \psi(w)) .
$$

Hereinafter, given a tuple of degree one, we denote the index of its only non-zero entry by $\left[\begin{array}{l}i_{\circ} \\ j_{0}\end{array}\right]$. Generalizing [29, Lemma 3.1], we obtain the following result. 
Lemma 2.10 Let $\left\{\left(e_{j}^{i}\right)^{\prime *} \mid 1 \leq i \leq n, 0 \leq j \leq d_{i}\right\}$ be the set of coordinate functions on the first copy of $B^{\prime}$ in (3) and $\left\{\left(e_{j}^{i}\right)^{\prime \prime * *}\right\}$ the respective set on the second one. ${ }^{1}$ Then

$\sec _{X}^{*}: z(c) \mapsto\left[\begin{array}{ll}t\left(e_{c_{j_{\circ}}^{i_{0}}}^{i_{\circ}}\right)^{\prime *}+(1-t)\left(e_{c_{j_{\circ}}^{i_{\circ}}}^{i_{\circ}}\right)^{\prime \prime *}, & \operatorname{deg} c=1 \text { with } c_{j_{\circ}}^{i_{\circ}} \neq 0, \\ t(1-t)(1-2 t)^{\operatorname{deg}(z(c))-2} \prod_{c_{j}^{i} \neq 0}\left(\left(e_{c_{j}^{i}}^{i}\right)^{\prime *}-\left(e_{c_{j}^{i}}^{i}\right)^{\prime / *}\right), & \operatorname{deg} c>1,\end{array}\right.$ for each $c \in C(\hat{A})$.

Proof Let $Y$ be the affine cone over the Segre product $\mathbb{P}\left(V_{1}\right)^{s_{1}} \times \cdots \times \mathbb{P}\left(V_{n}\right)^{s_{n}}$. Then the secant of $Y$ is parameterized by

$$
\sec _{Y}: \mathbb{A}^{1} \times B \times B \rightarrow \hat{A},(t, v, w) \mapsto \pi(t \tilde{\psi}(v)+(1-t) \tilde{\psi}(w)) .
$$

Thus, $\sec _{X}=\sec _{Y} \circ(\mathrm{id} \times e \times e)$. The statement follows after applying [29, Lemma 3.1] to $\sec _{Y}$.

\section{Torus action}

We can infer the following decomposition of $\sec _{X}$.

Notation 2.11 Let rep: $\mathbb{A}^{1} \times B^{\prime} \times B^{\prime} \rightarrow \mathbb{A}^{1} \times B^{\prime} \times B^{\prime}$, be a reparameterization:

$$
\text { rep: }(t, v, w) \mapsto\left(\frac{t(1-t)}{(1-2 t)^{2}}, t v+(1-t) w,(1-2 t)(w-v)\right)
$$

and let $m: \mathbb{A}^{1} \times B^{\prime} \times B^{\prime} \rightarrow \hat{A}$, be a monomial map:

$$
m^{*}: z(c) \mapsto\left[\begin{array}{ll}
\left(e_{c_{j_{\circ}}^{i_{\circ}}}^{i_{\circ}}\right)^{\prime *}, & \operatorname{deg} c=1 \text { with } c_{j_{\circ}}^{i_{\circ}} \neq 0, \\
t \prod_{c_{j}^{i} \neq 0}\left(e_{c_{j}^{i}}^{i}\right)^{\prime *}, & \operatorname{deg} c>1 .
\end{array}\right.
$$

Lemma 2.12 There is a decomposition $\sec _{X}=m \circ$ rep. In particular, $m$ is a monomial parameterization of $\operatorname{Sec} X$.

Proof Straightforward.

This monomial parameterization of $\operatorname{Sec} X$ already provides a structure of a toric variety on $\operatorname{im}(m)=\hat{A} \cap \operatorname{Sec} X$, hence provides us with a toric chart of $\operatorname{Sec} X$. Below we describe in detail its structure.

Notation 2.13 Let us introduce the following closed subsets of $\hat{A}$ :

$$
\begin{aligned}
\hat{A}_{1} & =\{z(c)=0 \mid \operatorname{deg} c>1\}, \\
\hat{A}_{2} & =\{z(c)=0 \mid \operatorname{deg} c=1\}, \\
S_{2} & =\operatorname{Sec} X \cap A_{2} .
\end{aligned}
$$

1 That is, $\left(e_{j}^{i}\right)^{\prime *}(v)=v_{j}^{i}$ and $\left(e_{j}^{i}\right)^{\prime \prime *}(w)=w_{j}^{i}$ respectively. 
Notation 2.14 (Lattice Polytope $P$ ) Consider the lattice $M=\bigoplus_{1 \leq i \leq n 1 \leq j \leq d_{i}} \mathbb{Z} \chi_{j}^{i}$, where $\chi_{j}^{i}=\left(e_{j}^{i}\right)^{*}$. Let $P \subset M \otimes \mathbb{Q}$ be the lattice polytope defined by inequalities

$$
\begin{cases}\left(\chi_{j}^{i}\right)^{*} \geq 0, & 1 \leq i \leq n, 1 \leq j \leq d_{i}, \\ \sum_{j=1}^{d_{i}}\left(\chi_{j}^{i}\right)^{*} \leq s_{i}, & 1 \leq i \leq n, \\ \sum_{\substack{1 \leq i \leq n \leq d_{i} \\ 1 \leq j \leq d_{j}}}\left(\chi_{j}^{i}\right)^{*} 2 . & \end{cases}
$$

Proposition 2.15 In the terminology above,

(i) Sec $X \cap \hat{A}=\hat{A}_{1} \times S_{2}$ via natural projections along coordinates $z(c)$,

(ii) $\hat{A}_{1}=X \cap \hat{A} \cong \mathbb{A}^{N}$, where $N=\sum_{i=1}^{n} \operatorname{dim} \hat{V}_{i}$,

(iii) $S_{2} \cong \operatorname{AffCone}\left(X_{P}\right)$, where $X_{P}$ is a projective toric variety with polarization corresponding to the polytope $P$, see, e.g., [11, §2.3] for the construction.

Proof The morphism $m$ is a direct product of $\left.m\right|_{\{0\} \times B^{\prime} \times\{0\}}$ and $\left.m\right|_{\mathbb{A}^{1} \times\{0\} \times B^{\prime}}$, which respectively parameterize $\hat{A}_{1}$ and $S_{2}$. This implies (i). The Segre-Veronese variety $X$ is also parameterized by $\left.m\right|_{\{0\} \times B^{\prime} \times\{0\}}$, thus (ii) holds.

Let us consider the standard torus $T=\operatorname{Spec} \mathbf{k}[M] \subset B^{\prime}$ and a projectivization $\pi_{z}: \hat{A} \backslash\{0\} \rightarrow \mathbb{P}(\hat{A})$ along $z(c)$-coordinates. Then $X_{P}=\overline{\pi_{z}\left(S_{2}\right)}$ is parameterized by a $T$-equivariant map $\left.\pi_{z} \circ m\right|_{\{0\} \times\{0\} \times B^{\prime}}$, which is defined by the set of monomials $\left\{\left(e_{c_{j}^{i}}^{i}\right)^{*} \mid\right.$ $\operatorname{deg} c>1\} \subset \mathcal{O}\left(B^{\prime}\right)$ corresponding exactly to lattice points $P \cap M$. This implies (iii).

Proposition 2.16 Let $\operatorname{Tan} X \subset \mathbb{P}(A)$ be the tangential variety of $X$. Then

$$
\operatorname{Tan} X \cap \hat{A}=\hat{A}_{1} \times X_{P}^{\prime},
$$

where $X_{P}^{\prime} \subset \hat{A}_{2}$ is a nondegenerate toric variety parameterized by $\left.m\right|_{\left\{-\frac{1}{4}\right\} \times\{0\} \times B^{\prime}}$.

Proof We present here a sketch of a proof for $\mathbf{k}=\mathbb{C}$, see [29] and [28, Lem. 3.3] for a complete proof. Consider $\left(\epsilon^{-1}, v, v+\epsilon w\right) \in \mathbb{A}^{1} \times B^{\prime} \times B^{\prime}$. If $\epsilon \rightarrow 0$, then $\sec _{X}\left(\epsilon^{-1}, v, v+\right.$ $\epsilon w)$ tends to an element of $\mathrm{T}_{\pi \circ \psi(v)} X \hookrightarrow$ Tan $X$ corresponding to $w$. On the other hand, by Lemma 2.10,

$$
\lim _{\epsilon \rightarrow 0} z(c)\left(\sec _{X}\left(\epsilon^{-1}, v, v+\epsilon w\right)\right)=\left[\begin{array}{ll}
v_{i_{i_{\circ}}}^{i_{\circ}}-w_{c_{j_{\circ}}^{i_{\circ}},}^{i_{\circ}}, & \operatorname{deg} c=1 \text { with } c_{j_{\circ}}^{i_{\circ}} \neq 0, \\
-\frac{1}{4} \prod_{c_{j}^{i} \neq 0} 2 w_{c_{j}^{i}}^{i}, & \operatorname{deg} c>1 .
\end{array}\right.
$$

Thus, the decomposition follows. It remains to check that $X_{P}^{\prime}$ is nondegenerate. Indeed, $\mathcal{O}\left(X_{P}^{\prime}\right) \hookrightarrow \mathcal{O}\left(B^{\prime}\right)$ does not contain invertible elements.

These propositions imply the following relationship of the tangential and secant varieties, which, in turn, implies Zak's theorem [40] for Segre-Veronese varieties.

Corollary 2.17 The following conditions are equivalent:

(i) $P$ is not contained in the hyperplane $\sum_{i, j}\left(\chi_{j}^{i}\right)^{*}=2$,

(ii) $\operatorname{dim} \operatorname{Sec} X=2 \operatorname{dim} X+1$,

(iii) $\operatorname{Sec} X \neq \operatorname{Tan} X$,

(iv) $\operatorname{dim} \operatorname{Tan} X=\operatorname{dim} \operatorname{Sec} X-1$. 
Then $\operatorname{Sec} X$ is called non-degenerate.

Proof Let $d=\operatorname{dim} X$. As a toric variety, $X$ is represented by a $d$-dimensional polytope $S$, which is a product of simplices. Then $P$ is the intersection of $S$ with the halfspace $\sum_{i, j}\left(\chi_{j}^{i}\right)^{*} \geq 2$.

(i) $\Rightarrow$ (ii) The assumption implies $\operatorname{dim} P=d$. Hence, $\operatorname{dim} S_{2}=d+1$ and $\operatorname{dim} \operatorname{Sec} X=$ $\operatorname{dim} \hat{A}_{1}+\operatorname{dim} S_{2}=2 d+1$.

$(i) \Rightarrow$ (iv) As before, $\operatorname{dim} \operatorname{Cone}(P)=d$. Hence, $\operatorname{dim} \operatorname{Tan} X=\operatorname{dim} \hat{A}_{1}+d=2 d$. The implications $(i i) \Rightarrow$ (iii) and (iv) $\Rightarrow$ (iii) are obvious.

(iii) $\Rightarrow$ (i) If $P$ is contained in the hyperplane, then all monomials corresponding to lattice points in $P$ are of the same degree. In particular, $X_{P}^{\prime} \cong \operatorname{AffCone}\left(X_{P}\right)$.

Example 2.18 1. Consider the Veronese surface $Y=\mathbb{P}^{2} \hookrightarrow \mathbb{P}^{5}$. It is a projective toric variety corresponding to the simplex $S=\operatorname{conv}\left(0,2 \chi_{1}, 2 \chi_{2}\right) \subset\left\langle\chi_{1}, \chi_{2}\right\rangle \cong \mathbb{Z}^{2}$, i.e. parameterized by characters of a two-dimensional torus that correspond to the lattice points of $S$. By Proposition 2.15, the variety $X_{P}$ that defines the secant is parameterized by $P=S \cap\left\{\chi_{1}^{*}+\chi_{2}^{*} \geq 2\right\}$, i.e., by the lattice points $2 \chi_{1}, \chi_{1}+\chi_{2}, 2 \chi_{2}$. Hence, both factors of $\operatorname{Sec} X \cap \hat{A}=\hat{A}_{1} \times S_{2}$ are of dimension two, so $\operatorname{dim} \operatorname{Sec} X=4$. Thus, the secant variety is degenerate and defined by $z(1,2)^{2}=z(1,1) z(2,2)$.

2. Consider the Segre product $\mathbb{P}^{1} \times \mathbb{P}^{1} \times \mathbb{P}^{1}$. The representing polytope is a cube $[0,1]^{3}$. The secant variety is represented by a polytope with vertices $(1,1,0),(1,0,1),(0,1,1)$, $(1,1,1)$. We see that the affine cone over it is the whole affine space; indeed, in this case the secant variety is non-degenerate and fills the whole ambient space. The tangential variety is a hypersurface defined by the equation $z(1,1,0) z(1,0,1) z(0,1,1)=z(1,1,1)^{2}$.

Theorem 2.19 The tangential and secant varieties of a Segre-Veronese variety are covered by complements of hyperplane sections. Each complement is an affine toric variety without torus factors. In case of the secant variety, these are always normal toric varieties. In case of the tangential variety they are normal if the underlying variety is the Segre product.

Proof By [39, Theorem 2.2] we know that $\operatorname{Sec} X$ is normal. By [29, Proposition 8.5] we know that Tan $X$ is normal, when $X$ is the Segre product. The open subsets $\operatorname{Sec} X \cap \hat{A}$ and Tan $X \cap \hat{A}$ are toric varieties by Propositions 2.15 and 2.16. Moreover, they do not contain torus factors, since they are products of an affine space $\hat{A}_{1}$ with either an affine cone over a projective toric variety $X_{P}$ or a non-degenerate toric variety $X_{P}^{\prime}$. By taking such subsets for various choices of basis vectors $e_{0}^{i}, i=1, \ldots, n$, we obtain the statement.

Theorem 2.20 Let $X=v_{s_{1}}\left(\mathbb{P}\left(V_{1}\right)\right) \times \cdots \times v_{s_{n}}\left(\mathbb{P}\left(V_{n}\right)\right)$ be a Segre-Veronese variety. Then the affine cone over the secant variety of $X$ is flexible. Further, if $s_{1}=\cdots=s_{n}=1$, then also the affine cone over the tangential variety of $X$ is flexible.

Proof By [5, Theorem 0.2] we know that all the affine charts from Theorem 2.19 are flexible. Now, by Theorem 1.4 we obtain flexibility of the affine cone.

Example 2.21 Consider the third Veronese embedding $X=v_{3}\left(\mathbb{P}^{1}\right)$. It is represented by characters in the interval $S=\operatorname{conv}(0,3)$. Then Tan $X \cap \hat{A} \cong \mathbb{A}^{1} \times X_{P}^{\prime}$, where the monoid of characters associated to the toric variety $X_{P}^{\prime}$ is generated by $\{2,3\}$. Namely, $X_{P}^{\prime}$ is the curve with a cusp singularity at the origin. Thus, Tan $X$ is a surface, whose singular locus is the curve $X$. This example can be generalized to tangential varieties of other Segre-Veronese varieties provided that at least one of the Veronese factors is of degree at least 3. 


\section{The combinatorial description of $T$-varieties}

We consider a normal variety $X$ with an effective action of an algebraic torus $T \cong\left(\mathbb{G}_{m}\right)^{r}$. Then $X$ is called a $T$-variety of complexity $(\operatorname{dim} X-\operatorname{dim} T)$. Here, the case of a complexityone torus is the most widely studied one, with contributions by many different authors [2, $16,21,24,35,37]$. In the following we restrict ourselves to the case of rational $T$-varieties of complexity one. Following [4] and generalising the classification of toric varieties by their fans, we introduce some combinatorial language to classify rational $T$-varieties of complexity one.

Let us denote the character lattice of the torus $T$ by $M$ and the dual lattice by $N$. For the associated $\mathbb{Q}$-vector spaces we write $M_{\mathbb{Q}}$ and $N_{\mathbb{Q}}$.

For a polyhedron $\Delta \subset N_{\mathbb{Q}}$ we define its tail cone as follows

$$
\operatorname{tail}(\Delta):=\left\{v \in N_{\mathbb{Q}} \mid \Delta+\mathbb{Q}_{\geq 0} \cdot v=\Delta\right\} .
$$

Now, we consider polyhedral complexes $\Xi$ in $N_{\mathbb{Q}}$. Here, by polyhedral complex we mean a set of convex polyhedra, which is closed under the face relation and every pair of polyhedra intersect in a common face. Moreover, we assume that the set of tail cones has the structure of a polyhedral complex itself, which is called the tail fan of $\Xi$ and will be denoted by tail $(\Xi)$. Consider a pair $\mathcal{S}=\left(\sum_{P \in \mathbb{P}^{1}} \mathcal{S}_{P} \otimes P, \operatorname{deg} \mathcal{S}\right)$ where $\mathcal{S}_{P}$ are polyhedral complexes in $N_{\mathbb{Q}}$ with some common tail fan $\Sigma$ and $\operatorname{deg} \mathcal{S} \subset|\Sigma|$. Here, $\sum_{P} \mathcal{S}_{P} \otimes P$ is just a formal sum. The complexes $\mathcal{S}_{P}$ are called slices of $\mathcal{S}$. We assume that there are only finitely many slices that differ from the tail fan $\operatorname{tail}(\mathcal{S}):=\Sigma$. The set of the points $P \in \mathbb{P}^{1}$ such that $\mathcal{S}_{P} \neq \Sigma$ is called the support of $\mathcal{S}$ and will be denoted by $\operatorname{supp} \mathcal{S}$. Note that for every full-dimensional $\sigma \in \Sigma$ there is a unique polyhedron $\Delta_{P}^{\sigma}$ in $\mathcal{S}_{P}$ with tail $\left(\Delta_{P}^{\sigma}\right)=\sigma$.

Definition 3.1 ( $f$-divisor) A pair $\mathcal{S}$ as above is called an $f$-divisor if for any full-dimensional $\sigma \in \operatorname{tail}(\mathcal{S})$ we have either $\operatorname{deg} \mathcal{S} \cap \sigma=\emptyset$ or

$$
\sum_{P} \Delta_{P}^{\sigma}=\operatorname{deg} \mathcal{S} \cap \sigma \subsetneq \sigma .
$$

An f-divisor as above corresponds to a rational $T$-variety of complexity one, see [19, Section 1]. Moreover, this correspondence is even functorial. In particular, invariant open subvarieties correspond to f-divisors $\mathcal{S}^{\prime}$, such that $\mathcal{S}_{P}^{\prime} \subset \mathcal{S}_{P}$ as sets of polyhedra and $\operatorname{deg} \mathcal{S}^{\prime}=$ $\left|\operatorname{tail}\left(\mathcal{S}^{\prime}\right)\right| \cap \operatorname{deg} \mathcal{S}$. For simplicity we write $\mathcal{S}^{\prime} \subset \mathcal{S}$ in this situation. As a consequence of Proposition 1.6 in [19], f-divisors $\mathcal{S}_{1}, \ldots, \mathcal{S}_{\ell} \subset \mathcal{S}$ give rise to an open covering if and only if their slices cover the slices of $\mathcal{S}$, i.e.

$$
|\mathcal{S}|=\bigcup_{i}\left|\mathcal{S}_{i}\right|
$$

Remark 3.2 Affine charts correspond to f-divisors $\mathcal{S}$ such that $\mathcal{S}_{P}$ consists of a single polyhedron (and its faces) and $\operatorname{deg} \mathcal{S}=\sum_{P \in \mathbb{P}^{1}} \mathcal{S}_{P}$. These objects are called p-divisors in [4,19].

Example 3.3 In Fig. 1 we sketched the non-trivial slices of an f-divisor as well as its degree. It describes the blowup of the quadric threefold in one point, see [33].

Lemma 3.4 [20, Remark 1.8.] Anf-divisor describes a subtorus action on a toric variety if and only if $\mathcal{S}_{P}$ equals a lattice translate of the tail fan for all but at most two $P \in \mathbb{P}^{1}$. 

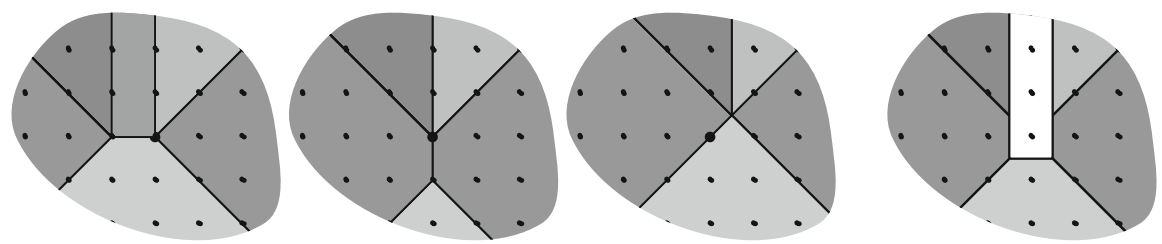

Fig. 1 An f-divisor

In the language of f-divisors we also may describe torus invariant Cartier divisors by support functions. A support function $h$ on a polyhedral subdivision $\Xi$ is a continuous function that is affine linear on every polyhedra in $\Xi$. We denote by $\operatorname{lin} h$ the linear part of $h$. This is a piecewise linear function on the tail fan defined as follows:

$$
(\operatorname{lin} h)(v):=h(w+v)-h(w)
$$

for some $w \in \Delta \in \Xi$ with $v \in \operatorname{tail}(\Delta)$.

Definition 3.5 (Support function on $\mathcal{S}$ ) A support function $h$ on an f-divisor $\mathcal{S}$ is a collection $\left\{h_{P}\right\}_{P \in \mathbb{P}^{1}}$ of support functions on $\mathcal{S}_{P}$ such that

(i) all $h_{P}$ have the same linear part, which will be denoted by lin $h$,

(ii) only finitely many of them differ from $\operatorname{lin} h$.

We have two kinds of torus invariant prime divisors on $X(\mathcal{S})$. Horizontal prime divisors correspond to rays $\rho \in \operatorname{tail}(\mathcal{S})^{(1)}$ that do not intersect $\operatorname{deg} \mathcal{S}$ and are denoted by $D_{\rho}$. Vertical prime divisors correspond to vertices $v$ in the subdivisions $\mathcal{S}_{P}$ and are denoted by $D_{P, v}$. Now, the divisor corresponding to the support function $h$ is given by

$$
D_{h}=-\sum_{\rho}(\operatorname{lin} h)(\rho) \cdot D_{\rho}-\sum_{P, v} \mu(v) \cdot h_{P}(v) D_{P, v},
$$

where we identify the ray with the ray generator and $\mu(v)$ denotes the minimal positive integer such that $\mu(v) \cdot v$ is a lattice element. In particular,

(i) if $h_{P} \leq 0$ for all $P \in \mathbb{P}^{1}$, then $D_{h}$ is effective.

(ii) in this case $X(\mathcal{S}) \backslash \operatorname{supp} D_{h}$ is given by the f-divisor

$$
[h=0]:=\left(\sum_{P}\left[h_{P}=0\right] \otimes P, \quad \operatorname{deg} \mathcal{S} \cap[\operatorname{lin} h=0]\right),
$$

where $\left[h_{P}=0\right]$ denotes the polyhedral subcomplex of $\mathcal{S}_{P}$ consisting of those polyhedra on which $h_{P}$ vanishes.

By [36, Section 4] (or [31]), every invariant Cartier divisor arises in this way. We have $D_{h} \sim D_{h^{\prime}}$ if and only if $h_{P}-h_{P}^{\prime}$ is affine linear for every $P$, i.e. $h_{P}-h_{P}^{\prime}=\langle u, \cdot\rangle+a_{P}$, and $\sum_{P} a_{P}=0$. Moreover, we have a criterion for ampleness expressed in the following notation. We denote by $\square_{h}$ the polytope given by

$$
\square_{h}=\left\{u \in M_{\mathbb{Q}} \mid\langle u, \cdot\rangle \geq(\operatorname{lin} h)\right\}
$$

and consider concave piecewise affine function $h_{P}^{*}$ on $\square_{h}$ as "dual" of $h_{P}$ :

$$
h_{P}^{*}(u):=\inf _{v}\left(\langle u, v\rangle-h_{P}(v)\right) .
$$

The definition implies that $h_{P}^{*}(u)$ is finite for $u \in \square_{h}$. 
Theorem 3.6 If $D_{h}$ is ample, then $h_{P}$ is strongly concave for every $P \in \mathbb{P}^{1}$ and $h_{P}^{*}(u) \geq 0$ for every $u \in \square_{h}$.

Proof By Petersen and Süß [31, Theorem 3.28], $h_{P}$ has to be strongly concave and by [19, Prop. 3.1(i)] we get that $h_{P}^{*}(u) \geq 0$.

Remark 3.7 On a $T$-variety every divisor is linearly equivalent to some torus invariant divisor. This follows for example from Fulton et al. [15, Theorem 1].

\section{Affine cones over projective $T$-varieties}

From now on we assume that the $T$-varieties which we consider are proper over the base, i.e. the corresponding f-divisors $\mathcal{S}$ satisfy the condition that all its slices $\mathcal{S}_{P}$ are subdivisions of $N_{\mathbb{Q}}$.

Definition 4.1 (Equivariant covering by toric charts) A $T$-variety is called equivariantly covered by toric charts, if there is an open covering by toric varieties $U_{i}$ such that the torus $T$ acts as a subtorus of the embedded torus of $U_{i}$.

Lemma 4.2 The $T$-variety $X(\mathcal{S})$ is equivariantly covered by toric charts if and only if for every maximal polyhedron $\Delta$ in $\mathcal{S}_{P}, P \in \mathbb{P}^{1}$, all but at most two slices contain a lattice translate of tail $(\Delta)$. In particular, either $X(\mathcal{S})$ itself is toric or there is at most one $P \in \mathbb{P}^{1}$ such that $\mathcal{S}_{P}$ does not contain a lattice vertex.

Proof The first part is a corollary of Lemma 3.4. To prove the last statement, we consider two points $P, Q$ such that $\mathcal{S}_{P}, \mathcal{S}_{Q}$ contain only non-lattice vertices. Now, consider a third point $R$ and a maximal polyhedron $\Delta \subset \mathcal{S}_{R}$. Since there is no lattice translate of tail $(\Delta)$ in $\mathcal{S}_{P}$ and $\mathcal{S}_{Q}, \Delta$ itself must be a translated cone. Notice that all maximal cones in $\mathcal{S}_{R}$ must be translated by the same lattice point. Indeed, otherwise they would not cover $N_{\mathbb{Q}}$ and there would exist a different maximal dimensional polyhedron that could not be a lattice translate of its tail cone. Hence, for any $R \notin\{P, Q\}$ the slice $\mathcal{S}_{R}$ is just a translated tail fan. By Lemma 3.4, $X(\mathcal{S})$ is a toric variety.

Remark 4.3 By [6, Appendix] this criterion is fulfilled for all smooth complete rational $T$ varieties of complexity one. Hence, they are covered by affine charts isomorphic to affine spaces.

To get flexibility for every affine cone we need to strengthen the condition in Lemma 4.2.

Theorem 4.4 Let $X=\mathfrak{X}(\mathcal{S})$ be a $T$-variety such that for any maximal polyhedron $\Delta \in \mathcal{S}_{y}$, $y \in \mathbb{P}^{1}$, at most two slices contain a polyhedron with the same tail cone tail $(\Delta)$ that is not a lattice translate of tail $(\Delta)$. Then for every very ample divisor $H$ the corresponding affine cone is flexible.

Proof By Theorem 1.4 it is enough to show that there exists a $T$-invariant $H$-polar covering by toric charts. Let us first rephrase this condition in terms of f-divisors. Remember that being $H$-polar means that the complement of every chart is the support of an effective divisor linearly equivalent to $H$. Having $T$-invariant charts means that we have to choose the effective divisors above to be $T$-invariant. Therefore, we are looking for a collection of support functions $h$, which via (4) give rise to divisors $D_{h} \sim H$, where $\sim$ denotes linear 
equivalence. Moreover, the open subsets $X \backslash \operatorname{supp} D_{h}$ have to cover $X$. By (5) the latter is equivalent to the fact that for every maximal polyhedron $\Delta_{P} \in \mathcal{S}_{P}$ there exists a strictly concave non-positive support function $h$ on $\mathcal{S}$ corresponding to an effective divisor $D_{h} \sim H$, such that $\left[h_{P}=0\right]=\Delta_{P}$ (i.e. $\left.h_{P}\right|_{\Delta_{P}} \equiv 0$ and negative elsewhere). For being a toric covering additionally we have to impose that $[h=0]$ has only two slices that are not lattice translates of the tail fan.

We now construct such a covering for some very ample divisor $H$. By Remark 3.7 every divisor is linearly equivalent to a torus invariant one. Hence, using [36, Section 4] we can assume that $H \sim D_{h}$ for some support function $h$. Fix a maximal polyhedron $\Delta \subset \mathcal{S}_{Q}$. Then $\left.h_{Q}\right|_{\Delta}$ is affine linear, i.e. $h_{Q}(v)=\langle u, v\rangle+a$. By concavity this implies $u \in \square_{h}$, with $\square_{h}$ defined as in (6). We now consider $h^{\prime}:=h-u$ with $h_{P}^{\prime}(v):=h_{P}(v)-\langle u, v\rangle$. Now, $h_{P}^{\prime}$ is again strongly concave and achieves its maximum at a polyhedron $\Delta_{P}$ with tail cone $\operatorname{tail}\left(\Delta_{P}\right)=\operatorname{tail}(\Delta)$. Moreover, by construction we have $0 \in \square_{h^{\prime}}$.

By our precondition, we may assume without loss of generality that for every point $R \in$ $\mathbb{P} \backslash\{0, \infty\}$ the polyhedron $\Delta_{R}$ is a lattice translate of tail( $\left.\Delta\right)$. Assume further $Q \neq \infty$ and introduce $h^{\infty}$ by

$$
\left\{\begin{array}{l}
h_{P}^{\infty}(v):=h_{P}^{\prime}(v)-\max \operatorname{Im} h_{P}^{\prime} \\
h_{\infty}^{\infty}(v):=h_{\infty}^{\prime}(v)+\sum_{P \neq \infty} \max \operatorname{Im} h_{P}^{\prime} .
\end{array} \quad \text { for } P \neq \infty,\right.
$$

It remains to check that $h_{\infty}^{\prime}(v)+\sum_{P \neq \infty} \max \operatorname{Im} h_{P}^{\prime} \leq 0$ to see that $D_{h^{\infty}}$ is indeed effective. Recall that we have $0 \in \square_{h^{\prime}}$. The claim follows from the ampleness of $D_{h^{\prime}}$ and Theorem 3.6.

Now, by construction we have $D_{h^{\infty}} \sim H$ and $\left[h_{Q}^{\infty}=0\right]=\Delta$. Moreover, $\left[h_{P}^{\infty}=0\right]$ is a lattice translate of tail $(\Delta)$ for each $P \notin\{0, \infty\}$. Then it describes a toric chart.

Taking these toric charts for every maximal polyhedron provides us with an $H$-polar covering. Now, our result follows by Theorem 1.4.

Theorem 4.5 All the affine cones over the Fano threefolds $Q, 2.29,2.30,2.31,2.32,3.8$, $3.18,3.19,3.23,3.24,4.4$, and certain elements of the families 2.24, 3.10 admitting a 2-torus action in Mori-Mukai's classification are flexible.

Proof For all Fano threefolds from Theorem 4.5 the corresponding f-divisors are listed in Süß [33]. One can easily check that the precondition of Theorem 4.4 is fulfilled in every case.

Example 4.6 Let us illustrate the difference of assumptions in Lemma 4.2 and Theorem 4.4. In the lemma we are allowed to choose the polyhedron with the given tail cone. Hence, if we consider the variety given by the slice $(-\infty,-1],[-1,1],[1, \infty)$ taken three times, then it does satisfy the assumptions. Indeed, if we take the maximal polytope $[-1,1]$ in one slice, in other two slices we can take just the vertex $\{1\}$, which is a lattice shift of the tail cone $\{0\}$. On the other hand, in the theorem we ask for all polyhedra with the given tail cone. Here, we get three times $[-1,1]$ which is not a lattice translate of $\{0\}$. Such a difference is only possible for cones that are not full-dimensional.

Example 4.7 We are coming back to the blowup of the quadric threefold from Example 3.3. We may check that the corresponding f-divisor in Fig. 1 fulfills the condition of Theorem 4.4. Hence, all the affine cones over the blowup of the quadric threefold are flexible.

Example 4.8 The hypersurface $\mathbb{V}\left(x_{0} y_{0}^{2}+x_{1} y_{1}^{2}+x_{2} y_{2}^{2}\right) \subset \mathbb{P}^{2} \times \mathbb{P}^{2}$ is 2.24 from our list in Theorem 4.5. Hence, every affine cone over this variety is flexible. In particular, this is true for the affine cone over the Segre embedding. 


\section{Total coordinate spaces}

We recall the definition of Cox rings.

Definition 5.1 (Cox sheaf, Cox ring, universal torsor, total coordinate space) Let $X$ be a complete normal variety, whose class group is a free abelian group. Assume that the classes of divisors $D_{1}, \ldots, D_{r}$ form a basis of this class group. The Cox sheaf of $X$ is defined by

$$
\mathcal{R}=\bigoplus_{\mathbf{a} \in \mathbb{Z}_{\geq 0}^{r}} \mathcal{O}\left(\sum_{i=1}^{r} a_{i} D_{i}\right) .
$$

It becomes a sheaf of $\mathcal{O}_{X}$-algebras via the usual multiplication of sections. The algebra $\mathcal{R}(X)$ of global sections of $\mathcal{R}$ is called the Cox ring of $X$.

The relative spectrum $\hat{X}=\operatorname{Spec}_{X}(\mathcal{R})$ is called the universal torsor of $X$. It is an open subset of the absolute spectrum $\bar{X}=\operatorname{Spec}(\mathcal{R}(X))$, which is called the total coordinate space of $X$. By construction, the Cox ring is graded by the class group of $X$ inducing an action of the torus $\operatorname{Spec} k[\mathrm{Cl}(X)]$ on the total coordinate space.

In the following we are studying flexibility of total coordinate spaces for several classes of varieties.

\subsection{Del Pezzo surfaces}

Since the smooth del Pezzo surfaces of degrees 6, 7, 8, and 9 are toric, their total coordinate spaces are just affine spaces and hence flexible. The remaining del Pezzo surfaces are blowups $X_{r}$ of $\mathbb{P}^{2}$ in $r$ points of general position, where $4 \leq r \leq 8$. Their Cox rings are described for example in Batyrev and Popov [8], Derenthal [13], and Testa et al. [38].

An exceptional curve on $X$ is a curve of self-intersection -1 and anti-canonical degree 1 . On every del Pezzo surface there are only finitely many of them, we denote their number by $N(r)$. Seen as an effective divisor every such curve $C$ corresponds to a section in the degree$[C]$ part of the Cox ring. This section is uniquely determined up to scaling by a non-zero constant.

We will use the following facts from Batyrev and Popov [8].

Theorem 5.2 [8, Thm 3.2 and Prop. 3.4] Let $N(r)$ be the number of exceptional curves on a del Pezzo surface $X_{r}$. Denote by $e_{1} \ldots, e_{N(r)}$ the sections corresponding to the exceptional curves and by $I$ the ideal of their relations. Then

(i) $\mathcal{R}\left(X_{r}\right)=\mathbf{k}\left[e_{1}, \ldots, e_{N(r)}\right] /$ I for $4 \leq r \leq 7$;

(ii) $\mathcal{R}\left(X_{8}\right)=\mathbf{k}\left[e_{1}, \ldots, e_{N(r)}\right] / I \oplus\left\langle f_{1}, f_{2}\right\rangle_{\mathbf{k}}$ as a vector space, where $f_{1}, f_{2} \in$ $H^{0}\left(X_{8}, \mathcal{O}\left(-K_{X_{8}}\right)\right)$ are elements of degree one with respect to the $\mathbb{Z}$-grading by the anti-canonical degree of a divisor class.

Theorem 5.2 shows that the Cox ring $\mathcal{R}(X)$ is generated by elements of degree 1 and $Y_{r}:=\operatorname{Proj}\left(\mathcal{R}\left(X_{r}\right)\right)$ comes with an embedding into $\mathbb{P}^{N-1}$, where $N$ (resp. $N-2$ ) is the number of exceptional curves in the case $4 \leq r \leq 7$ (resp. $r=8$ ).

In this situation the total coordinate space $\bar{X}_{r}$ is the affine cone over this embedding.

Proposition 5.3 Let e be a section corresponding to an exceptional curve. Then the principal open subset $\left(Y_{r}\right)_{e}$ is isomorphic to $X_{r-1}$.

Proof This can be found for example in the proof of Proposition 3.4 in Batyrev and Popov [8]. 
Theorem 5.4 The total coordinate spaces of smooth del Pezzo surfaces are flexible.

Proof As said above, it is enough to check the statement for $X_{r}$ with $4 \leq r \leq 8$. We will go by induction. The del Pezzo surface $X_{3}$ is toric. Therefore, it has a flexible total coordinate space. Now, consider $X_{r}$ with $4 \leq r \leq 8$. Then we have seen that $\bar{X}_{r}$ is the affine cone over $Y_{r}$. Moreover, the principal open subsets corresponding to sections of exceptional curves are isomorphic to $\bar{X}_{r-1}$ and hence flexible by induction hypothesis. It remains to check that these principal open subsets cover $Y_{r}$ to conclude flexibility of $\bar{X}_{r}$ from Theorem 1.4. For $4 \leq r \leq 7$ this follows directly from Theorem 5.2(i). For the case $r=8$ we have to take care for the remaining generators. By Theorem 5.2(ii) their squares are contained in the ideal $\left(e_{1} \ldots, e_{N}\right)$ generated by the sections corresponding to exceptional curves, but then the common vanishing of $e_{1} \ldots, e_{N}$ implies the vanishing of the remaining generators and hence $Y_{r}=\bigcup_{i=1}^{N}\left(Y_{r}\right)_{e_{i}}$.

\subsection{Smooth complexity-one $T$-varieties}

In Hausen and Süß [18] the Cox rings of $T$-varieties are studied. For the case of a complexityone action they have a very particular form.

Proposition 5.5 [18, Corollary 4.9] Let $\mathcal{S}$ be an $f$-divisor and let us denote by $\mathcal{S}^{\times}$the subset of rays in tail $(\mathcal{S})^{(1)}$ that do not intersect $\operatorname{deg} \mathcal{S}$. Then the Cox ring of $X(\mathcal{S})$ is given by

$$
\frac{\mathbf{k}\left[S_{\rho}, T_{P, v} \mid \rho \in \mathcal{S}^{\times}, P \in \operatorname{supp} \mathcal{S}, v \in \mathcal{S}_{P}^{(0)}\right]}{\left\langle z \cdot T^{\mu(0)}+T^{\mu(\infty)}+T^{\mu(z)} \mid z \in \operatorname{supp} \mathcal{S} \cap \mathbf{k}^{*}\right\rangle}
$$

where $T^{\mu(P)}:=\prod_{v \in \mathcal{S}_{P}^{(0)}} T_{P, v}^{\mu(v)}$ and $\mu(v)$ denotes the minimal positive integer such that $\mu(v) \cdot v$ is a lattice element.

If we impose the additional condition that the $T$-variety is equivariantly covered by toric charts (which is fulfilled in the smooth case), then we can conclude the following.

Proposition 5.6 The Cox ring of a complexity-one T-variety equivariantly covered by toric charts is isomorphic to

$$
\mathbf{k}\left[S_{1}, \ldots, S_{n_{S}} ; T_{\ell, j} \mid 0 \leq \ell \leq m, 1 \leq j \leq n_{\ell}\right] /\left\langle z_{\ell} \cdot A_{0}+A_{1}+A_{\ell} \mid 2 \leq \ell \leq m\right\rangle,
$$

where

(i) $N, m, n_{0}, \ldots, n_{m} \in \mathbb{Z}_{>0}$;

(ii) $z_{2}, \ldots, z_{m}$ are distinct elements of $\mathbf{k}^{*}$;

(iii) for $\ell=0, \ldots, m, A_{\ell}$ is a monomial in $\mathbf{k}\left[T_{\ell, 1}, \ldots, T_{\ell, n_{\ell}}\right]$;

(iv) for $\ell=1, \ldots, m$ the monomial $A_{\ell}$ is linear in at least one variable.

Moreover, if $X$ is Fano, then we may assume that $A_{\ell}$ for $\ell \geq 3$ is linear in each variable.

Proof The first statements follow directly from Proposition 5.5 and Lemma 4.2. For the Fano case note that the Cox ring of a Fano variety is log-terminal by Brown [9] and Gongyo et al. [17] and factorial by [7]. Hence, by Remark 6.4 in [25] we obtain the last statement.

Proposition 5.7 Let $X$ be a complexity-one $T$-variety equivariantly covered by toric charts and $\bar{X}$ be the total coordinate space of $X$. Then $\bar{X}$ is flexible. 
Proof Let $\mathbf{k}[\bar{X}]$ be as in Proposition 5.6. We assume that $\bar{X}$ is naturally embedded into an affine space $\mathbb{A}^{N}=\operatorname{Spec} \mathbf{k}\left[S_{1}, \ldots, S_{N} ; T_{\ell, j} \mid 0 \leq \ell \leq m, 1 \leq j \leq n_{\ell}\right]$.

The images of monomials $A_{0}, \ldots, A_{m}$ in $\mathbf{k}[\bar{X}]$ span a two-dimensional subspace, and no two of them are collinear. Therefore, we may permute $A_{0}, \ldots, A_{m}$ along with a proper change of their coefficients, indices of variables, and numbers $z_{i}$.

Lemma 5.8 The point $x \in \bar{X}$ is singular if and only if there are at least three monomials $A_{i}$ such that all their partial derivatives are vanishing at $x$.

Proof Let $x$ be singular and denote $L_{\ell}=z_{\ell} \cdot A_{0}+A_{1}+A_{\ell}$ for $\ell=2, \ldots, m$. Then there is a non-trivial linear combination $L \in\left\langle L_{2}, \ldots, L_{m}\right\rangle_{\mathbf{k}}$, whose partial derivatives vanish at $x$. Since $L$ is a sum of at least three monomials $A_{i}$, whose partial derivatives also vanish, the statement follows.

Conversely, given three monomials with partial derivatives vanishing at $x$, we assume that they are $A_{0}, A_{1}, A_{2}$. Then we take $L=L_{2}$.

So, for a smooth point $x \in X$, up to permutation of monomials and variables we assume that each monomial $A_{i}, i=2, \ldots, m$, has a non-zero partial derivative, say, $\frac{\partial A_{i}}{\partial T_{i, 1}}(x) \neq 0$. Moreover, we may choose $T_{i, 1}$ to be linear in $A_{i}$. Indeed, if $T_{i, 1}$ is not linear, then $A_{i}(x) \neq 0$, and we take $T_{i, 1}$ to be a linear variable by 5.6 (iv). We denote $B_{i}=\frac{\partial A_{i}}{\partial T_{i, 1}}$, which is non-zero at $x$.

Given a set of arbitrary numbers $c_{0,1}, \ldots, c_{0, n_{0}}, c_{1,1}, \ldots, c_{1, n_{1}} \in \mathbf{k}$, we construct a $\mathbb{G}_{a^{-}}$ action $\phi$ on $\mathbb{A}^{N}$ in two steps. First, denoting a parameter of $\mathbb{G}_{a}$ by $t$, we let

$$
\begin{aligned}
\phi^{*}: \mathbf{k}\left[\mathbb{A}^{N}\right] & \rightarrow \mathbf{k}\left[\mathbb{A}^{N}\right] \otimes \mathbf{k}[t], \\
S_{i} & \mapsto S_{i}, \quad \text { for } i=1, \ldots, n_{S}, \\
T_{0, j} & \mapsto T_{0, j}+t c_{0, j} \prod_{k=2}^{m} B_{k}, \quad \text { for } j=1, \ldots, n_{0}, \\
T_{1, j} & \mapsto T_{1, j}+t c_{1, j} \prod_{k=2}^{m} B_{k}, \quad \text { for } j=1, \ldots, n_{1} .
\end{aligned}
$$

Then, for some $H_{0}, H_{1} \in \mathbf{k}\left[\mathbb{A}^{N}\right] \otimes \mathbf{k}\left[\mathbb{G}_{a}\right]$,

$$
\begin{aligned}
& \phi^{*}\left(A_{0}\right)=A_{0}+H_{0} \prod_{k=2}^{m} B_{k}, \\
& \phi^{*}\left(A_{1}\right)=A_{1}+H_{1} \prod_{k=2}^{m} B_{k} .
\end{aligned}
$$

Now, for each $\ell=2, \ldots, m$ we let

$$
\begin{aligned}
& T_{\ell, 1} \mapsto T_{\ell, 1}-\left(z_{\ell} \cdot H_{0}+H_{1}\right) \prod_{\substack{2 \leq k \leq m \\
k \neq \ell}} B_{k}, \\
& T_{\ell, j} \mapsto T_{\ell, j}, \quad \text { for } j=2, \ldots, n_{\ell} .
\end{aligned}
$$

Then the trinomial $z_{\ell} \cdot A_{0}+A_{1}+A_{\ell}$ is fixed by $\phi^{*}$, so $\phi$ preserves $\bar{X} \subset \mathbb{A}^{N}$. Thus, we have constructed a $\mathbb{G}_{a}$-action on the total coordinate space $\bar{X}$, which we also denote by $\phi$. 
As said before, for a chosen smooth point $x$ we have $B_{i}(x) \neq 0$. Let us take another smooth point $y \in \bar{X}$ with non-zero coordinates and move $x$ to $y$ by $\mathbb{G}_{a}$-actions, denoting images of $x$ by same letter. By translations along coordinates $S_{1}, \ldots, S_{n_{S}}$ we 'equalize' them, i.e., obtain $S_{i}(x)=S_{i}(y), i=1, \ldots, n_{S}$.

Since $c_{0,1}, \ldots, c_{0, n_{0}}, c_{1,1}, \ldots, c_{1, n_{1}} \in \mathbf{k}$ are arbitrary, with $\phi$ we also equalize coordinates $T_{0, j}, j=1, \ldots, n_{0}$, and $T_{1, j}, j=1, \ldots, n_{1}$, at $x$ and $y$. Now, let $T_{1,1}$ be a linear variable in $A_{1}$, then for each $\ell=2, \ldots, m$ we construct a $\mathbb{G}_{a}$-action $\phi_{\ell}$ by permuting monomials $A_{1}$ and $A_{\ell}$ and applying the procedure above. Since $\frac{\partial A_{1}}{\partial T_{1,1}}(x)=\frac{\partial A_{1}}{\partial T_{1,1}}(y) \neq 0$, with $\phi_{\ell}$ we may equalize coordinates $T_{\ell, 2}, \ldots, T_{\ell, m}$, but break the equality of coordinate $T_{1,1}$, which we restore with $\phi$.

Proceeding in this way for each $\ell=2, \ldots, m$, we equalize all coordinates except $T_{2,1}, \ldots, T_{m, 1}$. But in this case the equation $z_{\ell} \cdot A_{0}+A_{1}+A_{\ell}=0$ with condition $B_{\ell}(x), B_{\ell}(y) \neq 0$ implies $T_{\ell, 1}(x)=T_{\ell, 1}(y)$ for each $\ell$. So, we may send any smooth point to any point with non-zero coordinates. Hence the action of SAut $\bar{X}$ is transitive on the regular locus of $\bar{X}$.

Theorem 5.9 The total coordinate space of a complete smooth rational $T$-variety of complexity one is flexible.

Proof By [6, Thm A.1], every rational smooth complete rational $T$-variety of complexity one is covered by affine spaces, so the statement follows from Proposition 5.7.

\subsection{Flexibility of total coordinate spaces vs. flexible coverings}

In [6] it was proved that for a variety with an open covering by affine spaces one obtains flexibility of the universal torsor. However, it is not clear whether the flexibility property extends to the total coordinate space. This motivates the following even more general question.

Question 5.10 Provided a variety admits an open covering by flexible affine subsets, does this imply flexibility of the total coordinate space?

It is also tempting to try to connect flexibility of the total coordinate space of the Cox ring of $X$ with that of affine cones over $X$. The following example shows that flexibility of the total coordinate space does not imply flexibility of all the affine cones.

Example 5.11 (Del Pezzo surfaces) We have seen in Sect. 5.1 that all total coordinate spaces of del Pezzo surfaces are flexible. On the other hand, del Pezzo surfaces are covered by affine spaces, which are flexible. Concerning flexibilty of affine cones it was shown in Perepechko [30] and Park and Won [32] that for degree 4 and 5 all the affine cones are flexible, but by Cheltsov et al. [23] and Kishimoto et al. [12] the anti-canonical affine cones over del Pezzo surfaces of degree 3,2, and 1 are not flexible.

One may still ask if flexibility of all the affine cones implies flexibility of the total coordinate space or for a more subtle relation, e.g. involving the grading of the Cox ring.

Question 5.12 Is there a relation between flexibility of the total coordinate space of $X$ and the fact that all affine cones over $X$ are flexible?

Let us give some illustrating examples for these questions.

Example 5.13 (Toric varieties) The Cox ring of a complete toric variety is a polynomial ring. Hence, the total coordinate space is flexible. On the other hand, the torus invariant affine charts and also the affine cones of a toric variety are again toric and hence flexible by Arzhantsev et al. [5, Theorem 0.2]. 
Example 5.14 (Blowups of a projective space in cubic hypersurfaces inside hyperplanes) The blowup constructions from Example 1.5 give varieties for which all the affine cones are flexible, as we have seen. On the other hand, the total coordinate space is flexible, as we see in the following.

We can consider the $k^{*}$-action on $\mathbb{P}^{n}$ given by multiplication with the coordinate $x_{0}$. It comes with a natural quotient map to $\mathbb{P}^{n-1}$ being defined outside the isolated fixed point $(1: 0: \ldots: 0)$. Then the centers of our blowups are fixed points of the action and we obtain induced actions on $X$ and $X^{\prime}$ with natural quotient maps given by composition of the original quotient map with the blowup. Now, we may use Theorem 1.2 in Hausen and Süß [18] to calculate the Cox rings

$$
\mathcal{R}(X) \cong \mathbf{k}\left[T_{0}, \ldots, T_{n}, T_{n}^{\prime}, S_{1}\right] /\left(T_{n} T_{n}^{\prime}-f\left(T_{0}, \ldots, T_{n-1}, 0\right)\right)
$$

and

$$
\left.\mathcal{R}\left(X^{\prime}\right) \cong \mathbf{k}\left[T_{0}, \ldots, T_{n}, T_{n}^{\prime}, S_{1}, S_{2}\right] /\left(T_{n} T_{n}^{\prime}-f\left(T_{0}, \ldots, T_{n-1}, 0\right)\right)\right) .
$$

We see that they are suspensions over an affine space and hence flexible by Theorem 0.2 in Arzhantsev et al. [5].

Example 5.15 ( $T$-varieties of complexity one) The proof of Theorem 5.9 implies that for $T$-varieties of complexity one the condition of being covered by toric (and hence flexible) charts is enough to deduce the flexibility of the total coordinate space. On the other hand, to conclude flexibility of all the affine cones we had to impose the stronger (technical) condition of Theorem 4.4.

Acknowledgements Open access funding provided by Max Planck Society. We would like to thank Mikhail Zaidenberg for motivating questions and inspiring results and Ivan Arzhantsev for many useful remarks and suggestions. The first author started the project under Mobilnosc+ Polish Ministry of Science program, finished under DAAD PRIME program and was supported by the Foundation for Polish Science (FNP). The formulation and proof of Lemma 1.1 (A.Perepechko) were supported by a Grant from the Dynasty Foundation. The research of A. Perepechko, which lead to the results of Sect. 5, was carried out at the IITP RAS at the expense of the Russian Foundation for Sciences (Project no. 14-50-00150).

Open Access This article is distributed under the terms of the Creative Commons Attribution 4.0 International License (http://creativecommons.org/licenses/by/4.0/), which permits unrestricted use, distribution, and reproduction in any medium, provided you give appropriate credit to the original author(s) and the source, provide a link to the Creative Commons license, and indicate if changes were made.

\section{References}

1. Arzhantsev, I., Flenner, H., Kaliman, S., Kutzschebauch, F., Zaidenberg, M.: Flexible varieties and automorphism groups. Duke Math. J. 162(4), 767-823 (2013)

2. Altmann, K., Hausen, J.: Polyhedral divisors and algebraic torus actions. Math. Ann. 334(3), 557-607 (2006)

3. Altmann, K., Hausen, J., Süß, H.: Gluing affine torus actions via divisorial fans. Transform. Groups 13(2), 215-242 (2008)

4. Altmann, K., Ilten, N., Petersen, L., Hendrik, V., Robert: The geometry of T-varieties. In: Pragacz, P. (ed.) Contributions to Algebraic Geometry: Impanga Lecture Notes (2011)

5. Arzhantsev, I., Kuyumzhiyan, K., Zaidenberg, M.: Flag varieties, toric varieties, and suspensions: three instances of infinite transitivity. Sb. Math. 203(7), 923-949 (2012)

6. Arzhantsev, I., Perepechko, A., Süß, H.: Infinite transitivity on universal torsors. J. Lond. Math. Soc. 89(3), 762-778 (2014)

7. Berchtold, F., Hausen, J.: Homogeneous coordinates for algebraic varieties. J. Algebra 266(2), 636-670 (2003) 
8. Batyrev, V., Popov, O.: The Cox ring of a del Pezzo surface. In: Arithmetic of Higher-Dimensional Algebraic Varieties. Proceedings of the Workshop on Rational and Integral Points of Higher-Dimensional Varieties, Palo Alto, CA, USA, December 11-20, 2002. Birkhäuser, Boston, pp. 85-103 (2004)

9. Brown, M.: Singularities of Cox rings of Fano varieties. J. Math. Pures Appl. 99(6), 655-667 (2013)

10. Ciliberto, C., Cueto, M., Mella, M., Ranestad, K., Zwiernik, P.: Cremona linearizations of some classical varieties. In: Gianfranco, C., Alberto, C., Letterio, G., Livia, G., Marina, M., Alessandro, V. (eds.) From Classical to Modern Algebraic Geometry, pp. 375-407. Springer International Publishing, Cham (2016)

11. Cox, D.A., Little, J.B., Schenck, H.K.: Toric varieties. Graduate Studies in Mathematics, American Mathematical Society, vol. 124, p. 841 (2011)

12. Cheltsov, I., Park, J., Won, J.: Affine cones over smooth cubic surfaces. J. Eur. Math. Soc. 18, 1537-1564 (2016)

13. Derenthal, U.: Universal torsors of del Pezzo surfaces and homogeneous spaces. Adv. Math. 213(2), 849-864 (2007)

14. Flenner, H., Kaliman, S., Zaidenberg, M.: A Gromov-Winkelmann type theorem for flexible varieties. J. Eur. Math. Soc. 18(11), 2483-2510 (2016)

15. Fulton, W., MacPherson, R., Sottile, F., Sturmfels, B.: Intersection theory on spherical varieties. J. Algebraic Geom. 4(1), 181-193 (1995)

16. Flenner, H., Zaidenberg, M.: Normal affine surfaces with $\mathbb{C}^{*}$-actions. Osaka J. Math. 40(4), 981-1009 (2003)

17. Gongyo, Y., Okawa, S., Sannai, A., Takagi, S.: Characterization of varieties of Fano type via singularities of Cox rings. J. Alg. Geom. 24(1), 159-182 (2015)

18. Hausen, J., Süß, H.: The Cox ring of an algebraic variety with torus action. Adv. Math. 225(2), 977-1012 (2010)

19. Ilten, N., Süß, H.: Polarized complexity-1 T-varieties. Mich. Math. J. 60(3), 561-578 (2011)

20. Ilten, N., Vollmert, R.: Deformations of rational T-varieties. J. Algebraic Geom. 21(3), 531-562 (2012)

21. Kempf, G., Knudsen, F., Mumford, D., Saint-Donat, B.: Toroidal embeddings I. Lecture Notes Math., vol. 339. Springer, Berlin (1973)

22. Kishimoto, T., Prokhorov, Y., Zaidenberg, M.: Group actions on affine cones. In: Montreal Centre de Recherches Mathématiques, CRM Proc. and lecture notes, vol. 54, pp 123-163 (2011)

23. Kishimoto, T., Prokhorov, Y., Zaidenberg, M.: Unipotent group actions on del Pezzo cones. Algebraic Geom. 1(1), 46-56 (2014)

24. Langlois, K.: Polyhedral divisors and torus actions of complexity one over arbitrary fields. J. Pure Appl. Algebra 219(6), 2015-2045 (2015)

25. Liendo, A., Süß, H.: Normal singularities with torus actions. Tohoku Math. J. 65, 105-130 (2013)

26. Mori, S., Mukai, S.: Classification of Fano 3-folds with $b_{2} \geq 2$. Manuscr. Math. 36(2), 147-162 (1981)

27. Masuda, K., Miyanishi, M.: Lifting of the additive group scheme actions. Tohoku Math. J. 61(2), 267-286 (2009)

28. Manivel, L., Michałek, M.: Secants of minuscule and cominuscule minimal orbits. Linear Algebra Appl. 481, 288-312 (2015)

29. Michałek, M., Oeding, L., Zwiernik, P.: Secant cumulants and toric geometry. Int. Math. Res. Not. 2015(12), 4019-4063 (2015)

30. Perepechko, A.: Flexibility of affine cones over del Pezzo surfaces of degree 4 and 5. Funct. Anal. Appl. 47(4), 284-289 (2013)

31. Petersen, L., Süß, H.: Torus invariant divisors. Isr. J. Math. 182, 481-505 (2011)

32. Park, J., Won, J.: Flexible affine cones over del Pezzo surfaces of degree 4. Eur. J. Math. 2(1), 304-318 (2016)

33. Süß, H.: Fano threefolds with 2-torus action-a picture book. Doc. Math. 19, 905-914 (2014)

34. Sturmfels, B., Zwiernik, P.: Binary cumulant varieties. Ann. Comb. 17(1), 229-250 (2013)

35. Timashev, D.A.: Classification of $G$-varieties of complexity 1. Izv. Math. 61(2), 363-397 (1997)

36. Timashev, D.A.: Cartier divisors and geometry of normal $G$-varieties. Transform. Groups 5(2), 181-204 (2000)

37. Timashev, D.: Torus actions of complexity one. Harada M. et al. (eds.) Toric topology. International conference, Osaka, Japan, May 28-June 3, 2006, Contemp. Math., vol. 460. American Mathematical Society (AMS), Providence, RI, pp. 349-364 (2008)

38. Testa, D., Várilly-Alvarado, A., Velasco, M.: Cox rings of degree one del Pezzo surfaces. Algebra Number Theory 3(7), 729-761 (2009)

39. Vermeire, P.: Singularities of the secant variety. J. Pure Appl. Algebra 213(6), 1129-1132 (2009) 
40. Zak, F.L.: Tangents and secants of algebraic varieties, volume 127 of Transl. Math. Monogr. American Mathematical Society, Providence, RI [Translated from the Russian manuscript by the author (1993)]

41. Zwiernik, P.: L-cumulants, L-cumulant embeddings and algebraic statistics. J. Algebraic Stat. 3(1) (2012)

42. Zwiernik, P.: Semialgebraic Statistics and Latent Tree Models. CRC Press, Boca Raton (2015) 